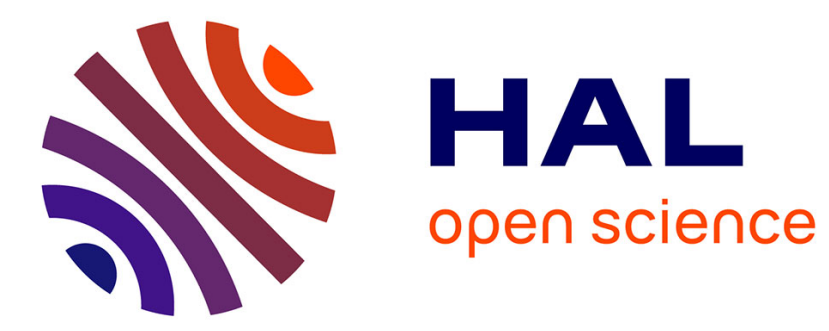

\title{
Les freins au travail collaboratif
}

Christine Gangloff-Ziegler

\section{To cite this version:}

Christine Gangloff-Ziegler. Les freins au travail collaboratif. Cahiers d'Economie et de Gestion de la Côte d'Opale. Le travail collaboratif, Une innovation générique, L'Harmattan, pp.95-112, 2009, Marché et Organisations. hal-00550661

\section{HAL Id: hal-00550661 https://hal.science/hal-00550661}

Submitted on 29 Dec 2010

HAL is a multi-disciplinary open access archive for the deposit and dissemination of scientific research documents, whether they are published or not. The documents may come from teaching and research institutions in France or abroad, or from public or private research centers.
L'archive ouverte pluridisciplinaire HAL, est destinée au dépôt et à la diffusion de documents scientifiques de niveau recherche, publiés ou non, émanant des établissements d'enseignement et de recherche français ou étrangers, des laboratoires publics ou privés. 


\title{
Les freins au travail collaboratif
}

\author{
Christine GANGLOFF-ZIEGLER
}

Si la notion de travail fait partie de l'histoire de l'humanité depuis ses origines, la question de son organisation c'est-à-dire, de sa répartition et de sa coordination au sein d'un groupe, s'est posée dès lors que les travailleurs se sont spécialisés. La collaboration et la coopération permettent de répondre à cette double exigence. Mais les concepts de travail collaboratif et de travail coopératif ont acquis une nouvelle dimension grâce aux technologies de l'information et de la communication, qui ont élargi ses potentialités puisqu'il est aujourd'hui possible de travailler ensemble, à une très grande échelle, sur un même projet, mais à distance, de manière synchrone ou asynchrone, sur des supports modifiables tout en permettant une relation directe entre les " collaborateurs " ou " coopérants " grâce à internet. A ce titre, le média qui détermine le mode de travail, transcende l'objet de la collaboration ou de la coopération tout en la rendant possible.

Mais, en soi, parler de travail collaboratif ou coopératif semble être un euphémisme dans la mesure où la notion de collaboration ou de coopération signifie étymologiquement " travailler ensemble ». Cependant, alors que ces deux notions envisagent uniquement l'action, le concept de travail collaboratif ou coopératif désigne à la fois le mode de contribution de chaque membre d'un groupe et le produit final issu de cette participation (Cord B., Ollivier C., 2001) identifiant ainsi simultanément l'action et la réalisation. II peut alors se définir comme le fait de travailler ensemble pour réaliser collectivement un projet. Mais, au-delà de cette définition commune et, malgré des racines latines proches, le travail collaboratif se différencie du travail coopératif. Etymologiquement, " operor » signifie travailler, effectuer, produire et "laboro" se traduit par travailler, se donner de la peine, le préfixe "co » supposant le fait d'agir ensemble et donc, nécessairement, à plusieurs. Par conséquent, entre opération et labeur, la notion de collaboration révèle une implication plus forte par la peine à laquelle elle fait référence.

Néanmoins, les auteurs ont cherché à identifier plus finement la distinction susceptible d'être faite entre le travail collaboratif et coopératif; et la distinction renvoie, comme nous l'indiquions précédemment, aux modes de répartition et de coordination du travail au sein d'un groupe, rendus nécessaires en raison d'une spécialisation croissante. «Toute activité humaine organisée (...) doit répondre à deux exigences fondamentales et contradictoires : la division du travail entre les différentes tâches à accomplir et la coordination de ces tâches pour l'accomplissement du travail. La structure d'une organisation peut être définie simplement comme la somme totale des moyens employés pour diviser le travail entre tâches distinctes et pour ensuite assurer la coordination nécessaire entre ces tâches » (Mintzberg, 2002).

Ainsi, « le travail coopératif est accompli par une division du travail dans laquelle chaque personne est responsable d'une partie de résolution d'un problème. La collaboration implique un engagement mutuel des participants dans un effort coordonné pour résoudre ensemble le problème. La distinction s'opère en distinguant les relations qu'entretient chaque individu avec les membres du groupe (obligation ou liberté) sa responsabilité par rapport aux actions (responsabilité déléguée au coordinateur 
ou constamment partagée) sa capacité à influer sur la définition et l'enchaînement des actions permettant d'atteindre l'objectif assigné au groupe (statut : hiérarchie ou égalité) » (Blanquet, 2007).

Nous en déduirons, en conséquence, que le travail coopératif se définit comme une forme d'organisation conjointe du travail où chacun des opérateurs est responsable pour sa part, parce qu'elle est identifiable, mesurable et prédéfinie par un coordinateur, responsable et représentant de l'autorité alors que le travail collaboratif est une forme d'organisation solidaire du travail où chacun est responsable pour le tout, sans que la part individuelle puisse être systématiquement isolée, la coordination se faisant par ajustement mutuel.

Certains auteurs conçoivent le travail collaboratif comme un nouveau modèle socio-économique de production, notamment lorsqu'il se développe en-dehors du cadre de l'entreprise et des marchés constituant ainsi des communautés qui produisent des œuvres intellectuelles, sans se référer à la notion de contrat et de propriété, d'égal à égal, entre pairs, sur un modèle dit de "peer-production » (Benkler, 2002). Mais, au-delà du partage de connaissances ou d'informations, «l'objet de la collaboration est de créer une vision partagée et des stratégies articulées pour faire émerger des intérêts communs dépassant les limites de chaque projet particulier »(Chrislip, 2002).

Nous évoquions précédemment l'essor que les technologies de l'information et de la communication ont apporté à cette forme de travail collectif. Elles proposent les outils du travail collaboratif qui peuvent se définir comme des outils de partage d'informations et de connaissances dans le but de réaliser collectivement un projet. Ceux-ci sont variés et évolutifs : messagerie différée ou instantanée, forums, partage de fichiers, de logiciels libres, de documents, webconférences, portail, annuaire commun, site partagé, wiki, moteur de recherche, agenda partagé ....

Séduisante en soi, la mise en place d'une organisation de travail collaborative rencontre néanmoins de nombreux freins qu'il convient d'identifier avant de pouvoir envisager les moyens permettant de les surmonter.

Notre questionnement consistera à déterminer quels sont les principaux obstacles au travail collaboratif pour reconnaître, a contrario, la présence des facteurs supposés de succès. Sur un plan méthodologique, nous avons observé les sites consacrés au travail collaboratif et, notamment, les échanges dans les forums; nous avons également exploité les résultats du réseau de recherche informel "Questions Expériences sur le Travail Collaboratif » ${ }^{1}$ dont nous faisons partie. Enfin, nous avons tiré les fruits de nos expériences, notamment sur la mise en place d'un site communautaire sur le pilotage de l'enseignement supérieur' ${ }^{2}$ et sur l'utilisation et le développement des outils intranet au sein de l'IUT de Colmar et de l'Université de Haute-Alsace (Lotus Notes et les outils collaboratifs associés, Google sites).

Mais, pour comprendre le modèle du travail collaboratif et pour en identifier les freins, il convient de déterminer au préalable le type de configuration qui le sous-tend.

\section{Le contexte organisationnel}

Dans une société du savoir, chère à P. Drucker (1993), le travail collaboratif peut s'analyser comme une forme d'intelligence collective fédérée autour d'un projet et reconfigurable selon l'évolution des objectifs. Un tel type d'organisation permet de surmonter la difficulté de la réalisation d'une œuvre commune complexe. Cette nouvelle forme de collaboration, médiatisée, se fonde essentiellement sur les compétences et dépasse les structures hiérarchisées et les systèmes organisationnels traditionnels qui en découlent.

Parmi les formes d'organisation décrites par Mintzberg (2002), nous retiendrons « l'adhocratie », appelée également organisation innovatrice, comme la forme de structuration la plus adaptée aux spécificités du travail collaboratif et adaptable aux différents types de projet, que leur objet soit lucratif ou non. Celle-ci présente une structure de type organique, correspond à un environnement évolutif et permet de répondre aux exigences d'un environnement complexe. En effet, pour s'adapter à un tel contexte, il faut pouvoir innover et « l'innovation sophistiquée requiert une configuration (...) capable de fusionner les travaux d'experts appartenant à des disciplines diverses dans des groupes de projets constitués en fonction des besoins et travaillant sans à-coup. » (Mintzberg, 2004) Celle-ci se coordonne par ajustement mutuel et donc par un échange entre les parties et repose sur des groupes de projet réunissant différentes compétences.

\footnotetext{
${ }_{2}^{1}$ http://www.qetc.info/wordpress/

2 www.wikisup.com
} 
La caractéristique principale de ce type de structure est de ne pas avoir de technostructure puisqu'il n'est pas envisageable de standardiser les procédés de travail, les savoirs ou les résultats. La composition des groupes varie selon les projets. II s'agit d'une organisation flexible destinée à répondre à la variabilité des marchés mais, c'est le projet, et non le marché ou la fonction, qui est fédérateur du groupe constitué. Le sommet stratégique n'est pas très développé et a surtout un rôle de coordination et de synthèse des travaux menés par les différents groupes. II définit également les règles communes de fonctionnement et peut jouer le rôle d'arbitre.

La coordination s'effectue par ajustement mutuel au sein des groupes mais également entre eux, ce qui n'exclut pas le conflit. Le savoir apparaît alors plus important que l'autorité et ce type d'organisation doit servir à assembler les différents savoirs de spécialistes, associés afin d'aboutir à la conception et à la réalisation d'un projet innovant et cohérent.

Si le travail collaboratif est d'évidence collectif, il est d'abord le résultat d'une somme d'interventions individuelles et des freins peuvent être identifiés à ces deux niveaux. Nous proposons alors de les sérier, autour de ces deux axes, en distinguant les facteurs individuels et les facteurs collectifs susceptibles de faire obstacle au travail collaboratif, afin d'en dresser un tableau aussi exhaustif que possible. En effet, il convient de construire un cadre susceptible de favoriser le travail collaboratif en suscitant une volonté de collaboration au sein de l'équipe ou de la communauté des collaborateurs ou contributeurs potentiels.

\section{Les freins individuels au travail collaboratif}

Nous avons précédemment défini le travail collaboratif comme une forme solidaire de travail. L'action est individuelle mais intégrée afin de réaliser une œuvre collective ; l'individu participe au résultat mais sans que sa participation puisse être identifiée, ni même mesurée. II s'agit en conséquence d'un mode de partage, partage des savoirs, des contributions et des résultats.

Parmi les facteurs individuels dont l'absence pourra constituer un frein au développement du travail collaboratif, nous nous distinguerons les aspects cognitifs ou volitionnels. Ces éléments présupposent cependant que les freins collectifs soient eux-mêmes levés.

Premièrement, les facteurs cognitifs supposent que les contributeurs aient les aptitudes requises dans ce cadre. "Les aptitudes peuvent être considérées (...) comme des caractéristiques cognitives responsables de l'acquisition et du traitement de l'information. " (Aubret, Gilbert, Pigeyre 1993) L'absence d'une aptitude peut ainsi déterminer l'échec de l'action pour laquelle cette aptitude est reconnue comme un préalable. Néanmoins, il est difficile d'identifier leur existence avant qu'elles ne se révèlent à travers les savoirs ou les actions.

Mais, si les savoirs renvoient habituellement à la cognition et l'expérience aux actions, dans la réalité la cognition et l'action sont nécessairement liées dans toute activité (Malglaive, 1990). Mais, elles le sont dans des proportions différentes selon que l'action est prescrite ou qu'elle impose de trouver de nouvelles réponses pour faire face à des évènements, au sens décrit par Zarifian (1999).

A la suite de Piaget (1970), nous soulignerons le fait que connaître ne consiste pas à copier le réel mais à agir sur lui et à le transformer. C'est alors cette capacité à agir sur le monde et à le changer, capacité exercée individuellement au service d'un collectif dont devront savoir faire preuve les acteurs concernés par le travail collaboratif.

Néanmoins, un facteur supplémentaire de complexité intervient puisque, au moins, deux types de savoirs se révèlent nécessaires : les savoirs liés à la spécialité du travail collaboratif et les savoirs procéduraux, nécessaires pour permettre le maniement des outils. L'insuffisance de ces derniers, liée à l'absence de formation et d'accompagnement, peut suffire à rebuter les individus, même s'ils ont les compétences requises dans la spécialité. Cette exigence de double compétence restreint le nombre des personnes susceptibles de participer à un travail collaboratif.

Mais, au-delà même de l'existence de ces savoirs, se pose la question de la représentation dont s'en font les contributeurs eux-mêmes. En effet, un des obstacles au travail collaboratif est le fait de ne pas se sentir légitime dans ses apports et/ou de redouter le regard critique des autres intervenants. Par exemple, un contributeur potentiel au développement d'un système d'exploitation ou d'un logiciel libre, comme par exemple Linux ou Open Office, peut craindre de générer un dysfonctionnement qui nuira à l'ensemble de la communauté et qui le désignera comme moins compétent que les autres au sein de celle-ci.

Nous remarquerons cependant que l'effet contraire peut aussi se produire. Si le niveau global des contributions est faible, l'intervenant peut craindre d'être assimilé à des personnes qui apparaîtraient 
moins expertes. II préférera alors se détourner de ce projet auquel il estimera dévalorisant de s'associer par rapport à l'image qu'il se fait de lui-même.

Deuxièmement, les facteurs volitionnels conduisent à s'interroger sur le processus conscient d'organisation des actions, dans le but d'atteindre délibérément l'objectif prédéfini. Ces facteurs amènent les individus à agir ou à s'abstenir après avoir traité les informations dont ils disposent. Ils sont ainsi amenés à faire des choix en fonction des opportunités et contraintes qu'ils rencontrent. Cela suppose, pour déclencher une action, que les intervenants appréhendent leur contribution dans un travail collaboratif, soit comme une contrainte à laquelle ils ne peuvent échapper, soit comme une opportunité qu'ils sont à même d'exploiter.

La contrainte peut découler de l'organisation du travail au sein d'une entreprise et de la subordination juridique liée au contrat liant un salarié et son employeur mais, moins le travail individuel est mesurable, moins il est facile de déterminer la part apportée par chacun et son degré d'implication. En conséquence, cela restreint l'intensité de la contrainte susceptible d'être exercée puisque certains vont être tentés de limiter leur apport en se conduisant comme des passagers clandestins, notion sur laquelle nous reviendrons dans le cadre de l'étude des freins collectifs au travail collaboratif. La contrainte peut également naître du caractère incontournable d'un réseau ou d'un outil qui impose son utilisation.

L'opportunité, quant à elle, apparaît dès lors que le contributeur estime qu'il trouvera un intérêt à collaborer, soit dans une perspective individualiste, soit une perspective altruiste. La perspective individualiste consiste à s'impliquer en attendant une forme de contrepartie, que ce soit en termes de rémunération, de reconnaissance sociale, d'intégration dans un groupe, de renforcement et/ou de valorisation de ses savoirs, d'avis critiques... II peut également s'agir d'une forme de jeu. La perspective altruiste suppose d'intervenir dans un travail collaboratif sans intérêt personnel direct, dans l'objectif philanthropique d'aider autrui. Cela relève alors du domaine du don (Pomian, Roche, 2002).

Mais, quelle que soit la perspective, la contrainte ou l'opportunité doit d'abord exister et, ensuite, être assez forte pour vaincre les obstacles récurrents qui se dressent face à la mise en œuvre d'une démarche collaborative.

Le manque de temps est, sans aucun doute, l'argument le plus courant mais il masque souvent d'autres réticences parfois plus difficilement avouables, et donc moins aisément identifiables.

Le travail collaboratif, en permettant de travailler à distance, modifie les pratiques antérieures qu'un salarié pourra chercher à conserver s'ils les percevaient comme des avantages : les déplacements s'il sont conçus comme un plaisir ou comme un élément participant à un statut social, la maîtrise de son temps à travers les agendas secrets (Brown, 2002) les échanges informels, les modes de rémunération ou les politiques de remboursement de frais, les systèmes de fidélisation qui procurent des avantages personnels...

Par ailleurs, l'utilisation d'outils collaboratifs peut être ressentie comme un frein dans certains cas, en rendant les actions trop transparentes ou structurées, ce qui constitue le revers d'une rationalisation que ces outils impliquent (Brown, 2002).

L'esprit individuel de compétition peut également constituer un obstacle majeur et il est, en effet, traditionnellement opposé à l'esprit de collaboration dans l'analyse des rapports de pouvoir au sein des organisations. Mintzberg (2004) a ainsi décrit les forces qui s'exercent sur les organisations en distinguant la compétition, où chacun tire de son côté, et la coopération, où tous vont dans le même sens, même s'il souligne qu'une combinaison des deux, qui serait profitable à l'organisation, peut être trouvée. Mais, l'esprit de compétition repose, à la fois, sur une image idéale de soi, sur un besoin de surpasser les autres, et sur un désir de reconnaissance, le plus souvent peu compatible avec une démarche collective où la contribution personnelle n'est pas identifiable et où, en conséquence, la valeur individuelle n'est pas mesurable.

De plus, la diffusion des informations et des savoirs par l'émetteur potentiel suppose qu'il les conçoive comme intéressantes ou utiles pour les destinataires; or, il ne les a pas nécessairement qualifiées comme telles. La définition de la nature, du niveau d'authenticité, d'originalité et de pérennité des informations susceptibles d'être partagées doit être mise en lien avec l'objectif de la collaboration envisagée.

Enfin, nous évoquerons la tentation protectionniste.

La transmission des connaissances peut se comprendre comme une perte de savoir puisqu'en devenant commun, il n'est plus distinctif. A ce titre, l'appréhension d'une perte du pouvoir lié à la détention d'informations et à la possibilité de décider de leur diffusion ou de leur rétention vis-à-vis de destinataires choisis, ne doit pas être négligée. Les détenteurs d'informations apparaissent comme les " sachants", et donc les référents, face à des prises de décision; et ils ne sont pas nécessairement 
prêts à renoncer à ce rôle qui les valorise, à leurs yeux. Ils peuvent, également, craindre de perdre une part de leur utilité au sein de l'organisation et de devenir, de ce fait, plus facilement interchangeables.

Dans un cadre ouvert, la mise à disposition de certains éléments peut faire craindre la perte des droits sur ces apports non clairement identifiés, mais il est cependant possible de protéger les écrits originaux au titre des droits d'auteur, avec des dépôts en ligne, afin de pouvoir faire la preuve de l'antériorité des écrits. Néanmoins, les simples idées, même si elles sont susceptibles d'être valorisées, ne sont pas protégeables à ce titre.

Pour terminer ce recensement des freins individuels du travail collaboratif, et toujours en lien avec la tentation protectionniste, le contributeur, notamment dans un cadre communautaire, est sensible à l'utilisation réelle ou supposée des données personnelles qu'il transmet dans ce cadre ainsi qu'à l'éventuelle utilisation commerciale des éléments de sa contribution.

Mais, au-delà de l'individu, certains freins apparaissent au niveau du groupe lui-même.

\section{Les freins collectifs au travail collaboratif}

Le travail collaboratif est une forme d'intelligence collective à l'origine d'une action de coconstruction d'un bien, d'un service, résultat d'une mise en commun de savoirs. Et, certains des freins au travail collaboratif se situent au niveau collectif.

Nous distinguerons successivement les freins dans un cadre entrepreneurial et dans un cadre communautaire.

Dans le cadre d'une entreprise d'abord, le choix d'une configuration organisationnelle en contradiction avec le modèle collaboratif peut être un facteur important d'échec. Nous avons déjà précisé à quel type de configuration organisationnelle correspondait plus particulièrement le travail collaboratif. A défaut, le risque est de mettre les intervenants face à des injonctions paradoxales : le respect de la hiérarchie, le contrôle des activités, la rétention d'informations, la réitération de solutions face aux exigences d'une organisation transversale fondée sur la confiance, sur le partage des informations et des savoirs, sur le travail par objectifs et sur la découverte de nouvelles solutions face aux « épreuves » (Pomian, Roche, 2002) qui supposent d'inventer de nouvelles solutions. Coleman (1976) faisait déjà référence à cette " mise à l'épreuve » comme facteur d'apprentissage par la confrontation des savoirs et de la réalité qui oblige à adapter les premiers à la seconde.

Or, dans le discours, « on a l'illusion d'un renouvellement radical des modes d'organisation du travail travail enrichi, poly-fonctionnalité, multivalence, travail en groupe- là où il n'y a le plus souvent que redistribution des tâches et des fonctions à l'intérieur de la force de travail » (Linhart, Malan, 1998). Mais, le travail prescrit limite fortement ou rend inutile l'activité de " régulation métacognitive » (Allal, SaadaRobert, 1992) qui conduit un sujet à évaluer, adapter ou faire évoluer son action pour atteindre le résultat.

Par définition, une organisation collaborative de travail repose sur l'acceptation d'une incertitude dans les procédures, mais aussi dans les résultats qui ne peuvent être standardisés comme ce peut être le cas dans une configuration de type bureaucratique, respectivement mécaniste ou professionnelle (Mintzberg, 2002). II faut donc trouver le bon degré d'autonomie (El Kadiri et al., 2008)

Et, plus il y a d'incertitude dans la réalisation d'un objectif défini, plus il faut favoriser l'initiative en raison des théories économiques de l'incomplétude du contrat et de la rationalité limitée des contractants qui soulignent l'impossibilité économique, intellectuelle et juridique de prévoir l'ensemble des facteurs nécessaires à l'exécution du contrat et donc de les intégrer expressément dans une convention.

L'employeur pourra dès lors rechercher, soit une capacité à obéir, soit une capacité à prendre des initiatives, selon qu'il construit une organisation mécaniste ou professionnelle, si nous nous en tenons aux deux formes d'organisations bureaucratiques. Ces capacités entretenues et développées sur une durée peuvent rendre difficile le passage d'un individu d'un type de structure à un autre, que ce soit dans un sens ou dans l'autre. L'obéissance réduit ou interdit les initiatives alors que l'initiative suppose une autonomie même relative de décision. Or l'initiative, par l'obligation de prendre des décisions réfléchies et justifiables a posteriori, face à un environnement changeant, est, sans aucun doute, un facteur essentiel d'apprentissage et d'entretien de l'intelligence puisque les solutions sont trouvées et non imposées. Cela rappelle la formule célèbre d'Albert Jacquard, "L'intelligence est un chantier perpétuel qui ne s'use que si on ne s'en sert pas. „ Et la routine induite par une organisation mécaniste laissant peu de place à la réflexion personnelle et à la nécessité de faire des choix peut réduire les capacités des individus qui ne sont pas mis en situation de devoir s'interroger et, par conséquent, de devoir comprendre. 
L'entreprise apparaît alors comme une entité en perpétuelle mutation, subie ou choisie, et elle devient à ce titre " un espace des possibles » où l'imprévu, l'incertain, le flou, l'urgence et les menaces ont leur part et qui « aboutit à désacraliser l'idée de rationalité absolue » (Fridenson, 2001).

II est, par conséquent, nécessaire de mettre en place des structures adaptatives qui permettent d'absorber progressivement les mutations pour assurer l'adaptation permanente des entreprises à un contexte mouvant. Mais « l'incertitude détruit la possibilité d'agir à partir d'une connaissance scientifique, rationnelle du monde. II ne reste plus, comme critères d'action, que l'opinion, la conviction et le jugement » (Reynaud, 1992) assis néanmoins sur les savoirs existants. Et, face à l'imprévisible et à l'incertain, nous constatons « le rôle croissant des connaissances qui ne sont cruciales qu'à un moment et dans un lieu donné » (Veltz, 2001).

Le Conseil européen de Lisbonne a lui-même souligné le fait que «construire l'économie de la connaissance la plus dynamique du monde, c'est faire reposer la compétitivité et la croissance sur le développement et la mise en valeur des capacités cognitives et professionnelles des personnes ॥ (2003).

II apparaît alors indispensable au sein de l'entreprise, d'une part, de disposer d'une réserve suffisante de connaissances actuelles et potentielles, d'autre part, de proposer un cadre favorisant leur expression, leur diffusion et leur mise en œuvre et, enfin, de faire de cette forme de travail collectif un objectif, affiché et partagé au sein de l'entreprise.

Enfin, la plupart des systèmes de management des ressources humaines reposent sur l'évaluation des capacités et de la performance individuelles et non collectives et ne sont pas donc pas adaptés à ce type de fonctionnement.

N'oublions pas cependant, au-delà des aspects organisationnels, stratégiques et managériaux, les freins financiers ou techniques, tels le manque de convivialité des outils proposés, leur complexité, leur disponibilité limitée, les restrictions apportées par les systèmes de sécurité mis en place, la capacité insuffisante des outils informatiques ou encore le coût élevé de certaines solutions proposées.

Dans un cadre communautaire ensuite, la différenciation repose essentiellement sur le caractère volontaire de la démarche collaborative. Mais, elle repose sur les mêmes exigences: liberté des membres, égalité entre eux même si leurs droits peuvent être différenciés concernant l'administration et la sécurité des outils, respect des contributions de chacun dès lors qu'elles correspondent à l'objectif défini et aux exigences légales. Les freins collectifs principaux sont liés à la nécessité de faire connaître le projet initié afin d'y faire adhérer un nombre important de personnes susceptibles d'y contribuer, sur le modèle, par exemple, de Linux, d'Open Office, de Wikipedia ${ }^{3}$ ou encore de Jurispedia ${ }^{4}$. Dans certains cas, la création de véritables communautés comme celle des Linuxiens permet de donner une identité qui renforce le groupe.

Cela renvoie à la loi de Metcalfe, applicable aussi bien dans un cadre entrepreneurial que communautaire qui souligne que l'utilité d'un réseau de communication est proportionnelle au carré du nombre des utilisateurs connectés au système $\left(\mathrm{N}^{2}\right)^{5}$. Bien que discutable et discutée, cette affirmation souligne l'idée que l'intérêt d'un réseau de travail collaboratif augmente d'une manière exponentielle avec le nombre de participants. C'est, en conséquence, la capacité à fédérer d'une communauté qui permet de la faire vivre et de la développer en la rendant incontournable.

II convient alors de lui donner du sens sur un plan collectif, et que chaque contributeur potentiel y trouve une signification sur le plan individuel, tant il paraît évident que l'intérêt de l'individu ne s'effacera jamais totalement au profit de l'intérêt d'un collectif.

Mais, le risque lié à l'existence de passagers clandestins (Olson, 1978) peut créer des réticences au sein de la communauté constituée. lls se définissent comme des membres d'un groupe qui vont se soustraire à l'effort collectif tout en cherchant à en bénéficier, en minimisant voire en évitant toute implication. Et, en contradiction avec la loi de Metcalfe, nous pouvons soulever le paradoxe d'Olson : « comme les groupes relativement petits sont fréquemment capables de s'organiser sur la base du volontariat et d'agir en conformité avec leurs intérêts communs et que les grands groupes ne sont pas dans l'ensemble en mesure d'y parvenir, l'issue du combat politique qui oppose les groupes rivaux n'est pas symétrique... Les groupes les plus petits réussissent souvent à battre les plus grands qui, dans une démocratie, seraient naturellement censés l'emporter "(Olson, 1978). En effet, plus un groupe sera important, moins la contribution marginale sera sensible, et plus les individus pourront être tentés de

\footnotetext{
${ }_{4}^{3}$ http://fr.wikipedia.org/wiki/Accueil

${ }_{5}^{4}$ http://fr.jurispedia.org/index.php/Accueil

5 La loi de Metcalfe a été qualifiée ainsi en 1993 par G. Gilder dans le Gilder Technological Report:

http://www.gildertech.com/pastbooks.html
} 
rester en retrait si le rapport entre le coût et le bénéfice individuels de l'action collective est ainsi plus favorable.

Pour conclure, nous avons cherché à recenser les principaux facteurs susceptibles de freiner le travail collaboratif. Ils peuvent être individuels ou collectifs et cela souligne la difficulté de mettre en place une organisation collaborative répondant aux attentes identifiées. Cette réflexion révèle surtout des entrelacs complexes composés d'éléments cognitifs, conatifs, organisationnels, juridiques, managériaux, techniques, financiers, économiques, sociaux, sociologiques, psychologiques... qui rendent nécessairement fragmentaire la compréhension de tous les ressorts du travail collaboratif. Cette difficulté est encore amplifiée par la grande variété des modalités et des outils du travail collaboratif.

Néanmoins, si leur caractérisation, même partielle, permet d'entrevoir les facteurs clés du succès ou de l'échec de ce mode d'organisation du travail, c'est leur combinaison qui est vraisemblablement déterminante de la réussite du processus. C'est à la modélisation de celle-ci qu'il pourra être utile de s'attacher demain.

\section{Bibliographie}

ALLAL L., SAADA-ROBERT M. (1992) La métacognition: cadre conceptuel pour l'étude des régulations en situations scolaires, Archives de Psychologie 60, 265-296.

AUBRET J., GILBERT P., PIGEYRE F. (1993) Savoir et pouvoir, Les compétences en question, PUF, Paris.

BENKLER Y. (2002) Coase's Penguin, or, Linux and The Nature of the Firm, http://www.benkler.org/CoasesPenguin

BLANQUET M.-F. (2007) Web collaboratif, web coopératif, web 2.0. Quelles interrogations pour l'enseignant documentaliste ? Formation des personnes ressources en documentation, CRDP d'AixMarseille, http://eprofsdocs.crdp-aix-marseille.fr/-Web-collaboratif-Web-cooperatif-.html.

BROWN S. (2002) «Pour tous les processus séquentiels, les outils collaboratifs ont un avenir, pour le reste... », Interview, JDN Solutions.

http://www.journaldunet.com/solutions/itws/021028_kpmg.shtml.

CHRISLIP D. (2002) The Collaborative Leadership Fieldbook, A Guide for Citizens and Civic Leaders, San Francisco: Jossey-Bass.

COLEMANJ.S. (1976) Differences between experiential and classroom learning, in Keeton M. (dir.), Experiential learning: Rational, characteristics and assessment, 49-61. San Francisco: Jossey-Bass.

CORD B., OLLIVIER C. (2001) En quoi le travail collaboratif peut être soutien à la recherche de cohérence dans un processus de construction d'un cadre d'enseignement/apprentissage du Français Langue Etrangère (FLE) sur internet? Actes des colloques « Usages des nouvelles technologies et Enseignement des Langues Etrangères ", La Bibliothèque de l'Université de Technologie de Compiègne, pp. 51-64.

DRUCKER P. (1993) Au-delà du capitalisme, Paris : Village mondial.

EL KADIRI S., PERNELLE P., DELATTRE M., BOURAZ A., (2008) Pilotage des processus collaboratifs dans les systèmes PLM, Quels indicateurs pour quelle évaluation des performances, 1er Congrès des innovations mécaniques CIM'08, Sousse : Tunisie.

http://hal.archives-ouvertes.fr/docs/00/33/82/37/PDF/SEIKadiri_CIM2008.pdf

FRIDENSON P. (2001) Les apports de l'histoire des entreprises, in Pouchet A. (sous la coordination de) Sociologies du travail, 40 ans après, Paris : Elsevier.

LINHART D., R., MALAN A. (1998) Syndicats et organisation du travail : un rendez-vous manqué, Revue Sociologie et sociétés, vol. XXX, n² 2, automne 1998, éd. Les Presses de l'Université de Montréal.

MALGLAIVE G. (1990), Enseigner à des adultes, Paris : PUF, coll. Education et Formation.

MINTZBERG H. (2002) Structure et dynamique des organisations, Paris : Editions d'Organisation.

MINTZBERG H. (2004) Le management, Voyage au centre des organisations, Paris: Editions d'Organisation.

OLSON M. (1978) Logique de l'action collective, Paris : PUF.

PIAGET J. (1970), L'épistémologie génétique, Paris: PUF.

POMIAN J., ROCHE C. (2002) Connaissance capitale - Management des connaissances et organisation du travail, Paris : L'Harmattan. 
Processus de Lisbonne (2003) Communication du bureau à partir des avis du conseil de l'Europe et en préparation du sommet de printemps 2004, Paris : Journaux Officiels, 1.12.2003.

REYNAUD B. (1992) Le salaire, la règle et le marché, Paris : Christian Bourgois, collection Cibles XXI, par référence à Knight F. (1921) Risk, incertainty and profit, Houghton Mifflin Company.

VELTZ P. (2001) La sociologie du travail peut-elle encore parler de technique ? in Pouchet A. (sous la coordination de) Sociologies du travail, 40 ans après, Paris : Elsevier.

ZARIFIAN P. (1999) Objectif compétence pour une nouvelle logique, Paris : Editions Liaisons. 